\title{
Green Coffea robusta (Coffea canephora) from Lampung province effect toward free radicals in chickens infected with Salmonella enteritidis bacteria
}

\author{
Dahliatul Qosimah" ${ }^{1 *}$, Djalal Rosyidi², Lilik E. Radiati², Indah A. Amri ${ }^{1}$, Dodik Prasetyo ${ }^{3}$, \\ Fajar S. Permata ${ }^{4}$ and Agri K. Anisa ${ }^{5}$ \\ ${ }^{1}$ Laboratory of Microbiology and Immunology, Faculty of Veterinary Medicine, Brawijaya University, Malang, Indonesia \\ ${ }^{2}$ Animal Product Technology Laboratory, Faculty of Animal Science, Brawijaya University, Malang, Indonesia \\ ${ }^{3}$ Animal Clinical, Faculty of Veterinary Medicine, Brawijaya University, Malang, Indonesia \\ ${ }^{4}$ Laboratory of Anatomy Pathology, Faculty of Veterinary Medicine, Brawijaya University, Malang, Indonesia \\ ${ }^{5}$ Laboratory of Pharmacology, Faculty of Veterinary Medicine, Brawijaya University, Malang, Indonesia
}

\begin{abstract}
Background: Foodborne diseases are caused by acquired pathogenic bacteria such as Salmonella enteritidis. It causes an intestinal imbalance and the microbial toxins found in the gastrointestinal tract induce symptoms such as diarrhea. Coffee contains active ingredients such as antioxidants and is used as an anti-inflammatory agent by reducing proinflammatory cytokine levels in the body.

Aim: The purpose of this study was to determine the interaction between Lampung's robusta coffee and tissue damage in chickens infected by S. enteritidis.

Methods: This study used first-day-old Isa brown layer chickens $(n=60)$, which were divided into five treatment groups. The negative control group consisted of healthy and normal chickens, whereas the positive control group consisted of chickens infected with S. enteritidis bacteria at a concentration of $10^{8} \mathrm{CFU} / \mathrm{ml}$. Groups T1, T2, and T3 were given coffee extract with doses of $500 \mathrm{mg} / \mathrm{kg} \mathrm{BW}$ (low dose), 1,000 mg/kg BW (moderate dose), and 1,500 mg/ kg BW (high dose), respectively, and then infected with S. enteritidis bacteria at a concentration of $10^{8} \mathrm{CFU} / \mathrm{ml}$. The coffee extract and bacteria were given orally via a feeding tube at a volume of $0.5 \mathrm{ml}$ per chick. The extract was given for 14 days (from day 3 to day 16), and the bacteria were given on days 16 and 17. On day 18, the chickens were necropsied. The malondialdehyde (MDA) level was analyzed using one-way analysis of variance test with the GLM procedure $(<0.05)$, while the tissue histopath was analyzed using a descriptive qualitative study to examine the ileal damage

Results: The results showed that the MDA levels (nmol/l) decreased in treatment groups T1, T2, and T3 compared to the positive control. On the contrary, we found improvements in the ileum histopathology of group T1 and T2 in the form of normal and regular intestinal epithelium arrangement of the ileum, long intestinal villi, and decreased total leukocytes.

Conclusion: Green coffee robusta has the potential to increase antioxidants and reduce inflammation in the small intestine of chickens infected with S. enteritidis.

Keywords: Layer chicks, Antioxidants, Green coffee, Enteric bacteria.

\section{Introduction}

Consumption of food contaminated with pathogenic microorganisms (or poison) at infective doses often causes outbreaks of foodborne diseases. Food poisoning due to pathogenic bacteria ingestion could cause intestinal balance disorders, and the microbial toxins produced could result in diarrhea (Joseph et al., 2016). Diarrhea is most frequently associated with Salmonella typhimurium and Salmonella enteritidis, which are the primary causative agents of acute gastroenteritis (Khan, 2014). The digestive tract (GIT) is an ecosystem is suitable for enteric bacterial pathogens because macro and micronutrients in the epithelial cell layer enable bacterial invasion (Omojate et al., 2014).

Coffee is one of the essential food commodities in the world's economy. The Coffea genus has more than 100 species, but Coffea arabica (arabica) and Coffea canephora (robusta) are the most used as commercial commodities (Dias and De, 2015). Coffee is a very popular drink consumed by the public and is a rich nonenzymatic source. It contains bioactive components such as caffeine, chlorogenic acid (CGA), trigonelline, cafestol, and kahweol, which at varying amounts act as a free radical activity or antioxidant activity (Liang and David, 2014). A proper physiological function requires a balance between free radicals and antioxidants. If free radicals exceed the body's ability to regulate it, a condition known as oxidative stress occurs,
\end{abstract}


especially in cell membranes containing fat called malondialdehyde (MDA) or lipid peroxidase. Free radicals will change fats, proteins, and DNA that trigger several diseases in humans and animals (Lobo et al., 2010). Endogenous and exogenous antioxidants act as "free radical scavengers" by preventing and repairing damage caused by reactive oxygen species (ROS) and reactive nitrogen species (RNS). Therefore, they can increase the immune system and reduce disease risk (Pham-Huy et al., 2008). Caffeic acid and trigonelline show inhibitory power against bacteria (MartinezTome et al., 2011).

To date, there has been no scientific study that states the function of coffee as antioxidants in chickens. This study aims to examine Lampung's coffee extract's effect as an anti-inflammatory agent in layer chicks infected with S. enteritidis.

\section{Materials and Methods}

\section{Animals}

A total of 60 1-day-old layer chicks (Isa brown) were divided into five treatment groups. Each group contained 12 chicks placed in cages.

\section{Experimental design}

Chickens were placed in closed cages, fed with commercially standard Charoen Pokphand 511-bravo, with nutritional contents containing rough protein $21 \%-23 \%$, fat $5 \%-8 \%$, coarse fiber $3 \%-5 \%$, ash $4 \%-7 \%$, and were free of antibiotics and coccidiostats. Chickens were also given commercial vitamins orally (mixed in drinks) at the age of 1,5 , and 11 days to prevent stress and help adapt to the environment. On day 4, the chicks were given live Newcastle disease (ND)-IB (Infectious bronchitis) vaccines, and on day 10 , they were given ND. G7B vaccines and influenza AI H5N1 subtypes.

The treatments consisted of the negative control (healthy and normal chicks), positive control (infected with $S$. enteritidis bacteria at a concentration of $10^{8}$ $\mathrm{CFU} / \mathrm{ml}$ ), T1, T2, and T3 (chicks given coffee extract at a dose of 500, 1,000 and 1,500 mg/kg BW, respectively, and infected with $S$. enteritidis bacteria). Coffee was given daily for 14 days, from day 3 to day 16. $S$. enteritidis bacteria was given at a concentration of 0.5 $\times 10^{8} \mathrm{CFU} / \mathrm{ml}$ for 2 days on day 16 and day 17 . Coffee extract and bacteria were given orally with a volume of $0.5 \mathrm{ml}$. On the 18th day, the chicks were slaughtered, blood samples were collected, and necropsy on the small intestine was performed.

\section{Preparation of coffee extract}

Lampung's Robusta coffee seed powder was extracted using $90 \%$ ethanol. The extraction process was carried out at UPT Materia Medika, Batu, Indonesia. A total of $414 \mathrm{~g}$ of coffee powder was soaked in 1,500 ml of $90 \%$ ethanol solution three times and shaken using a shaker at speed, and then the filtrate was collected. The filtrate was evaporated using a rotatory evaporator. The coffee's active ingredient was tested using liquid chromatography-mass spectrometry (LC-MS) and phytochemical test (Mir et al., 2016) to detect the presence of phyto compounds.

Qualitative phytochemical examination showed that the coffee extract contains alkaloids and tannins, while the LC-MS tests showed that the extract contains 128.65 $\mu \mathrm{g} / \mathrm{mg}$ CGA (Kinuthia et al., 2017). The experiment used the brown extract obtained from this process. The coffee extract was given orally via a gastric tube at a volume of $0.5 \mathrm{ml}$.

\section{Preparation of the bacterial suspension}

Salmonella enteritidis bacteria were planted in the nutrient broth media and incubated at $37^{\circ} \mathrm{C}$ for 24 hours. The density of growing bacteria was then measured using the McFarland 0.5 standard (prepared by mixing $0.5 \mathrm{ml}$ of $1.75 \%(\mathrm{~b} / \mathrm{v})$ barium chloride dihydrate $\left(\mathrm{BaCl} 2.2 \mathrm{H}_{2} \mathrm{O}\right)$ with $99.5 \mathrm{ml} 1 \%(\mathrm{v} / \mathrm{v})$ sulfuric acid $\left(\mathrm{H}_{2} \mathrm{SO}_{4}\right)$. The estimated bacterial concentration used is $1-2 \times 10^{8} \mathrm{CFU} / \mathrm{ml}$ (Gayathiri et al., 2018). The bacterial suspension was given orally with a volume of $0.5 \mathrm{ml}$ per chick using a feeding tube on day 16 . Clinical symptoms were observed until day 18 . On day 18 , the chickens were slaughtered by neck decapitation, and necropsy was carried out (Qosimah et al., 2020).

\section{Determination of antioxidant activity}

Blood samples $(1.5 \mathrm{ml})$ were taken via the brachial vein before slaughter. EDTA anticoagulant was added to the blood sample, and serum samples were used to test MDA. MDA, an indicator for lipid peroxidation, was measured using the thiobarbituric acid (TBARS) method. TBARS was added to the supernatant, and 100 $\mu \mathrm{l}$ serum sample was heated at $90^{\circ} \mathrm{C}$ for 90 minutes. The supernatant's absorbance was measured at $532 \mathrm{~nm}$ wavelength (Afshordi et al., 2016) with a UV-visible spectrophotometer. MDA levels are indicated as nmol/l.

\section{Histopathological observation}

At the end of the study, necropsy was carried out on the chicks using the air embolism technique through the foramen magnum. Ileum tissue samples were collected and put into $10 \%$ formalin solution, followed by dehydration up to the final stage using paraffin. Tissue samples were then mounted to object glasses and stained using hematoxylin eosin (Mohamed et al., 2014).

\section{Data analysis}

MDA levels were analyzed using the one-way analysis of variance (ANOVA) test with a confidence level of 0.95 . Data with a significant difference were separated using orthogonal polynomial contrast tests. Histopathology was analyzed using a descriptive and qualitative study to determine ileal damage.

\section{Ethical approval}

The Brawijaya University's ethical committee approved all procedures in the study (Ethical Clearance No. 773-KEP-UB).

\section{Results}

The results of the MDA level measurement using T. acid (TBARS) are presented in Table 1. The average MDA 
Table 1. Orthogonal contrast test of MDA level.

\begin{tabular}{lc}
\hline Treatment & MDA level (nmol/l) \\
\hline Negative control (normal) & $1.54 \pm 0.2^{\mathrm{a}}$ \\
Positive control (Concentration of S. enteritidis, $\left.10^{8} \mathrm{CFU} / \mathrm{ml}\right)$ & $3.17 \pm 0.3^{\mathrm{c}}$ \\
T1 (coffee extract $500 \mathrm{mg} / \mathrm{kg}$ bw+concentration of $S$. enteritidis, $\left.10^{8} \mathrm{CFU} / \mathrm{ml}\right)$ & $2.46 \pm 0.2^{\mathrm{b}}$ \\
T2 (coffee extract $1,000 \mathrm{mg} / \mathrm{kg}$ bw+concentration of $S$. enteritidis $\left.10^{8} \mathrm{CFU} / \mathrm{ml}\right)$ & $2.81 \pm 0.3^{\mathrm{bc}}$ \\
T3 (coffee extract $1,500 \mathrm{mg} / \mathrm{kg}$ bw+concentration of $S$. enteritidis $\left.10^{8} \mathrm{CFU} / \mathrm{ml}\right)$ & $2.55 \pm 0.6^{\mathrm{bc}}$ \\
p-values for contrast & 0.000 \\
Linear & 0.005 \\
Quadratic & 0.000 \\
\hline
\end{tabular}

a,b,c Different superscripts indicate levels of significance at $p \leq 0.05$.
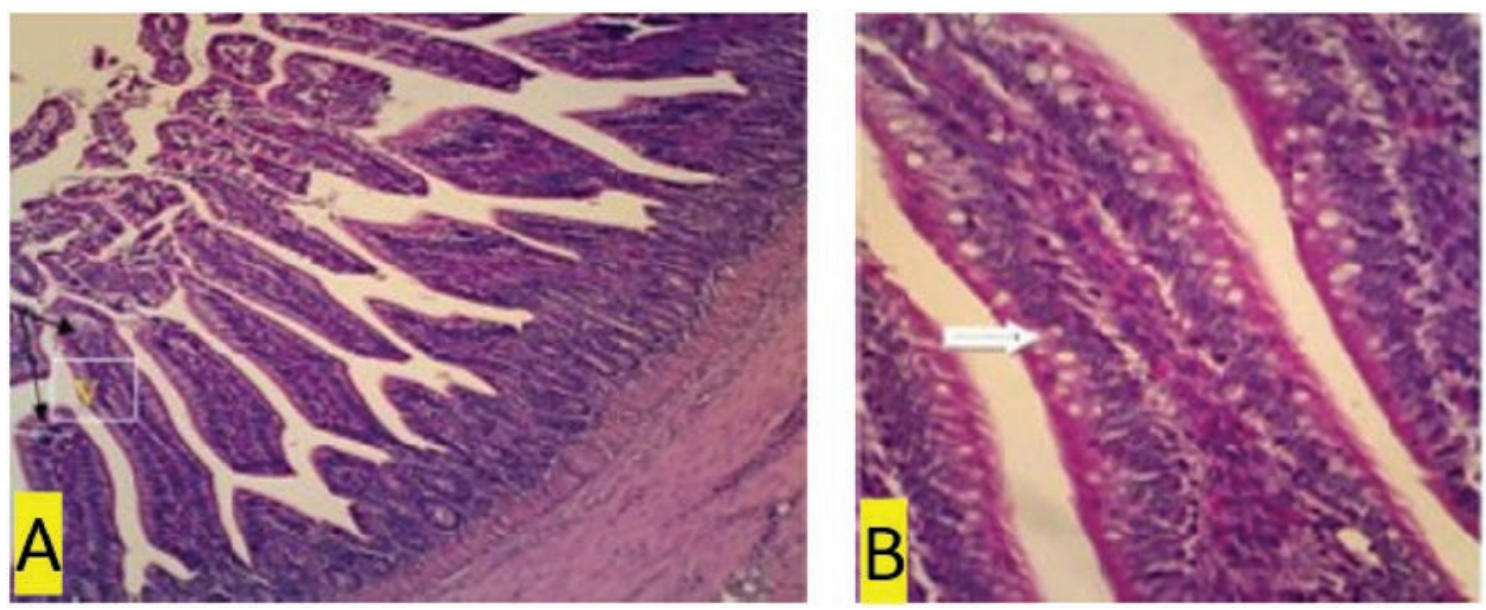

Fig. 1. (a) Negative control. In the ileal organs appear normal villi (V) (black arrow) (100x magnification). (b) Negative control. In the ileum organ appear normal goblet cells (white arrow) of epithelial regular arrangement.

level of the positive control group was significantly higher compared to the negative control. While in the T1, T2, and T3 groups, the average MDA levels decreased compared to the positive control, especially in the T2 group, which showed a significant difference. However, the average MDA levels of the treatment groups T1, T2, and T3 were still significantly higher than that of the negative control.

\section{Histopathology observation}

This study showed that compared to the negative control and treatment groups T1 and T2 (Figs. 1b, 3b and $4 \mathrm{~b}$ ), the positive control group (chickens infected with S. enteritidis bacteria) and T3 had erosions in the ileum (Figs. 2b and 5b). The negative control and treatment groups T1 and T2 showed normal epithelial arrangement with elongated villi (Figs. 1a, 3a, and 4a), and many goblet cells (Figs. 1b, 3b, and $4 \mathrm{~b}$ ). While in the T3 treatment group, we observed epithelial erosion and short vili resembling that of positive control treatment (Figs. 2a and 5a).

The intestinal villi's ability to absorb nutrients can be examined using morphometric calculations, including villi height (VH), villi width (VW), villus surface area, and the crypt depths. Villus morphometry can be observed using an Olympus microscope equipped with an X21 projector (camera) OptiLab and arranged with a magnification of 40 times. Villus heights are measured from the tip of the villi to the base, excluding crypts, and villus widths are measured halfway between the villus base and the tip, and the crypt depths are measured from the villus base upward to the region of transition between the crypt and villus (Hossain et al., 2014). According to the research conducted by Moghaddam and AlizadehGhamsari (2013), the villus surface areas are calculated using the formula: $2 \times 3.14 \times(\mathrm{VW} / 2) \times \mathrm{VH}$.

\section{Discussion}

The MDA levels (nmol/l) of the chicks in the negative control (C1) group (healthy chickens) were lower in the sick chicks [positive control (C2)]. MDA is a secondary lipid peroxidation product that acts as a marker to indicate the level of lipid oxidation. It can also act as a second messenger or react directly with biomolecules to disrupt biochemical processes (Lobo et al., 2010). MDA reacts with DNA to cause DNA damage. Lipid peroxidation refers to oxidative lipid degradation. It 

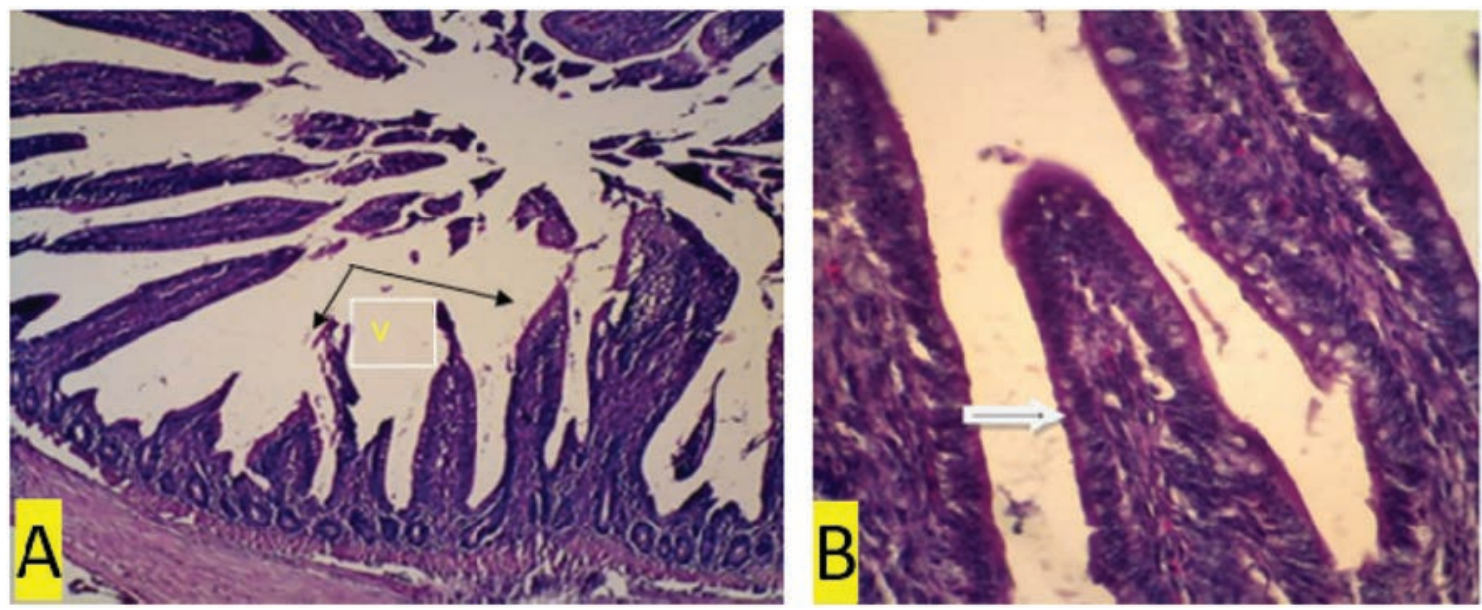

Fig. 2. (a) Positive control. In the ileum organ appear shortened villi (V) (black arrow) (100x magnification). (b) Positive control. 400x magnification. In the ileal organ epithelial erosion, little goblet cells (white arrows).
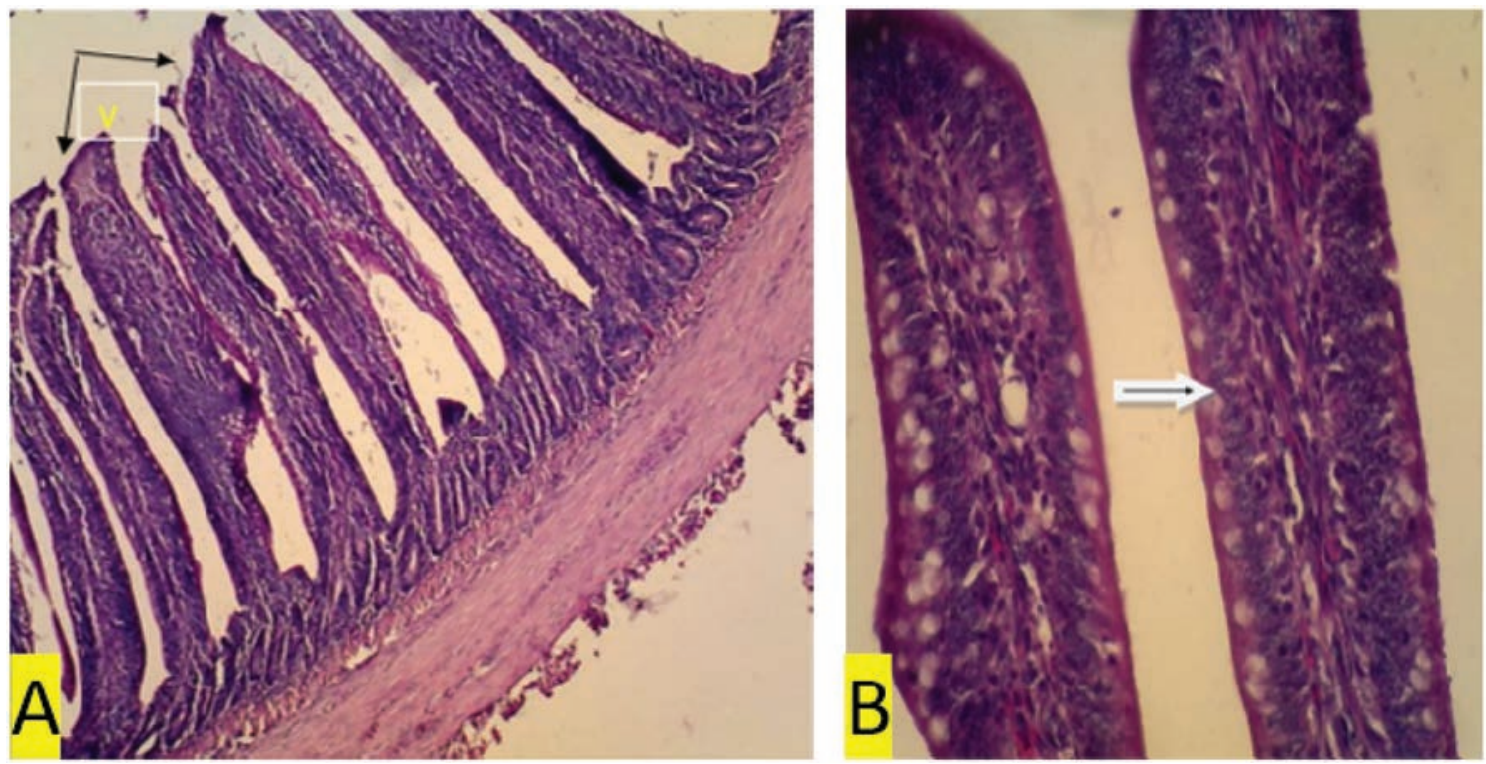

Fig. 3. (a) T1 treatment. In organ ileum villi appear higher than normal treatment (V) (black arrow) (100x magnification). (b) T1 treatment. Regular and normal arrangement of epithelium (white arrow).

usually affects polyunsaturated fatty acids because they contain multiple double bonds between the methylene bridges $(-\mathrm{CH} 2-)$, which have a reactive hydrogen. Salmonella enteritidis bacteria can produce high MDA concentrations and cause oxidative damage (Afshordi et al., 2016). Oxidative damage to membranes changes the degree of membrane fluidity, which can cause impaired membrane integrity, inactivation of membrane-bound receptors and enzymes, thus impairing normal cellular function, and increasing tissue permeability. Furthermore, lipid peroxidation's final product can act as a "second cytotoxic messenger" to induce cell damage. Some of these products readily react with various biomolecules, including proteins,
DNA, and phospholipids, to produce a stable product at the end of the reaction series thought to contribute to the disease's pathogenesis (Aprioku, 2013).

The average MDA level was the lowest in the negative control group and is significantly different from that of the positive control and the $\mathrm{T} 1, \mathrm{~T} 2$, and $\mathrm{T} 3$ treatment groups, as shown in Table 1 . Homogeneity requirements using Levene's test was homogenous at 0.419 ( $p>$ 0.05). Thus, the contrast option of GLM orthogonal polynomial was used. Based on the ANOVA test, there are differences in the average levels of MDA between treatment groups. Treatment using coffee extracts in the feed of chickens infected with $S$. enteritidis bacteria, in a linear, quadratic, and fourth manner can reduce 

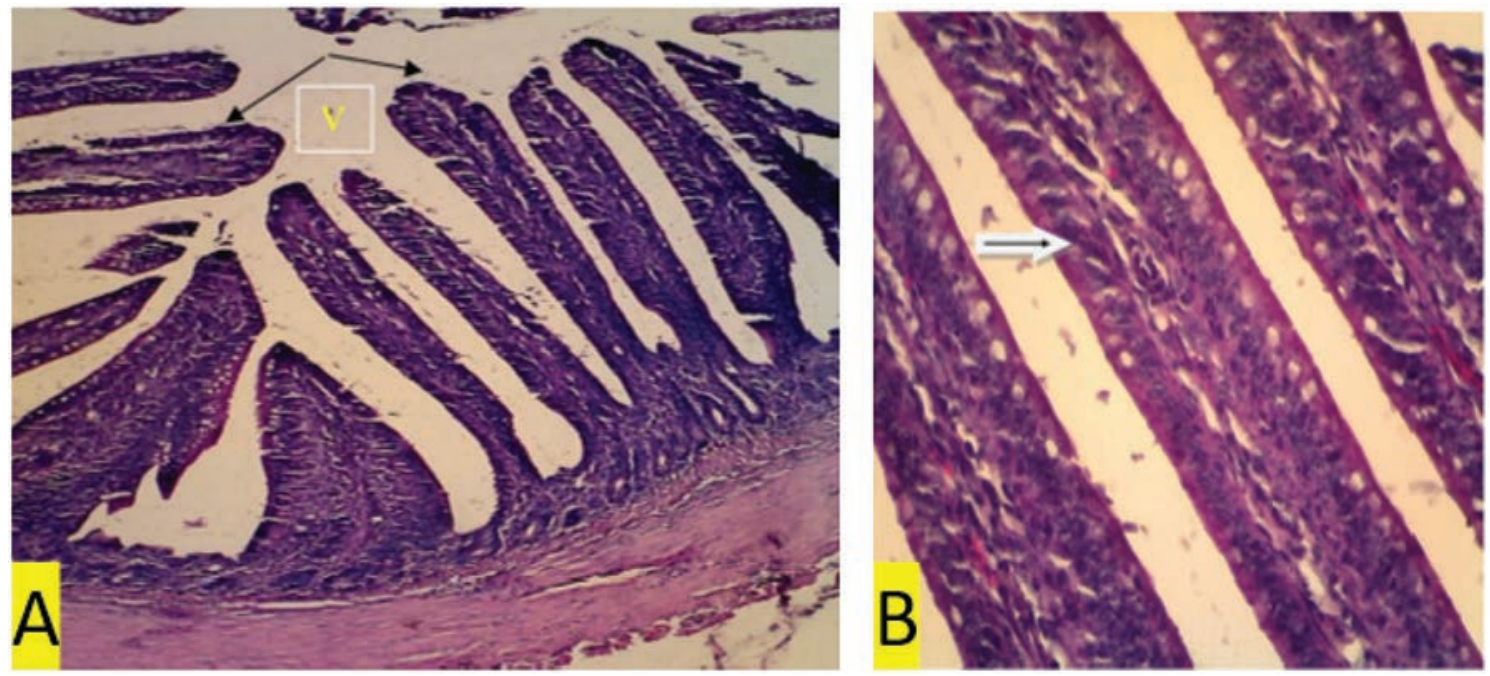

Fig. 4. (a) T2 treatment. In the ileum organ appear long villi (V) (black arrow) (100x magnification). (b) T2 treatment. In the ileum organ, epithelial cell regeneration appears, and the nucleus is many. Regular and normal epithelial arrangement and many goblet cells (white arrows) (400x magnification).
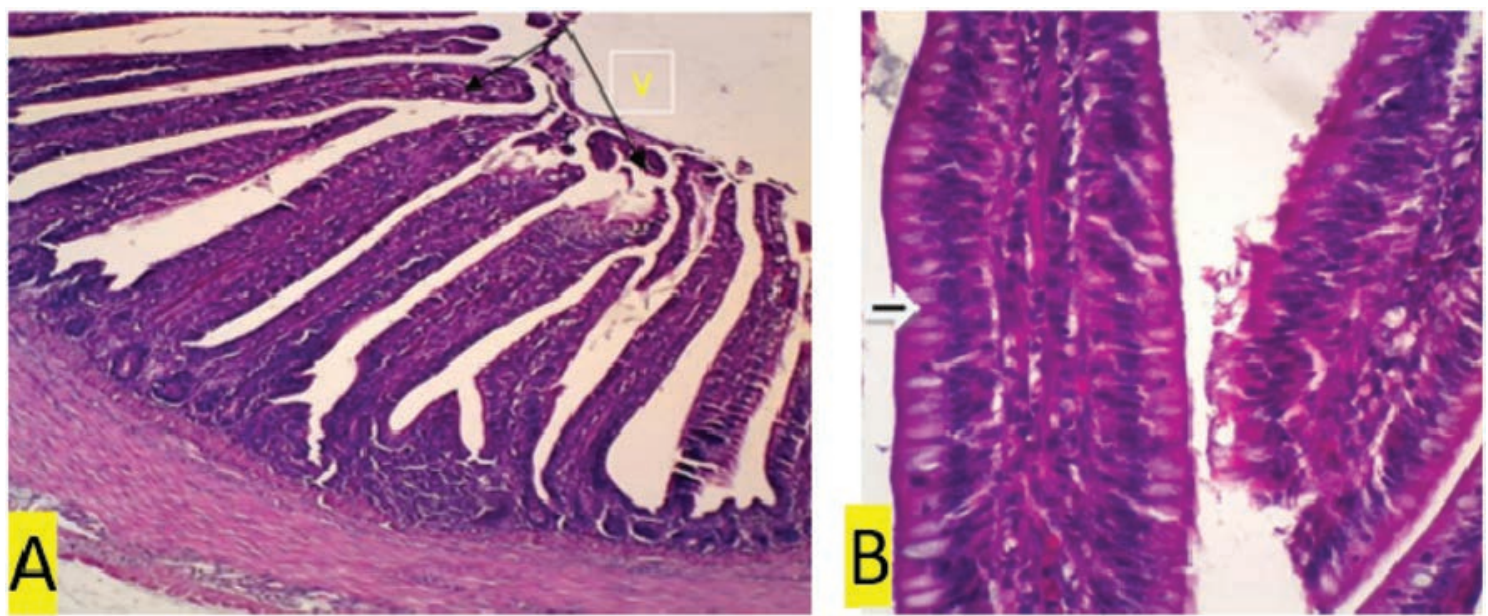

Fig. 5. (a) T3 treatment. In the ileal organ erosion epithelium appears (100x magnification). (b) T3 treatment. In the ileum organ seemed little so that the epithelial goblet cells did not function properly (400x magnification).

MDA levels ( $p=0.005, p=0.000$, and $p=0.002$, respectively). The administration of low-dose coffee extract decreased the MDA level at a higher magnitude than that of high dose $(p=0.000)$.

Salmonella bacteria infect the host through contaminated food and drink. After digestion, Salmonella will react with an acidic stomach and intestinal $\mathrm{pH}$. It has a tolerance toward acid. After entering the small intestine, Salmonella is exposed to the destructive effects of the luminal contents, including bile salts, enzymes, antimicrobial peptides, and IgA secretory. It has different infection pathways, i.e., through M-cells, intestinal epithelial cells, phagocytic cells, or across damaged intestinal barriers. After it penetrates the epithelial cell barrier, it is engulfed by intestinal macrophages and induces systemic disease (Khan, 2014). Inflammation of the intestines also decreases the absorption of nutrients and consequently results in weight loss (Mishra and Jha, 2019).

The GIT microbiota consists mainly of bacteria and protozoa. The microbiota population varies throughout the GIT compartment, most of which are found in the distal segment. In response to commensal bacteria, the intestinal epithelium produces ROS, which functions as the second messenger and cellular signal activation. Intestinal epithelial cells function to prevent the invasion of microorganisms. Mucosal interactions with microbes or their toxins trigger oxidative stress. Oxidative stress in cells or tissues results from an imbalance between free radical production and endogenous antioxidant 
defenses that cause lipid peroxidation, protein nitration, DNA damage, and apoptosis. Cells will be continuously exposed to free radicals produced during physiological oxygen metabolism. ROS and RNS at certain levels show molecules involved in homeostasis. The crossing proteins between two adjacent epithelial cells and luminal intestinal epithelium cell to prevent paracellular absorption of toxins. Oxidative stress changes cellular processes and functions as an intestinal barrier (Mishra and Jha, 2019). The presence of pro-inflammatory cytokines can be used to detect specific inflammation in the tissues.

Compared to the positive control, coffee extract administration reduced the MDA level of S. enteritidisinfected chickens. However, the condition of those chickens was still not comparable to that of healthy chickens (negative control) that were not infected with $S$. enteritidis. These results proved that the extract of local Indonesian coffee, Robusta Lampung, has an antioxidant potential against infectious agent caused by bacteria.

Microorganisms have mechanisms to protect themselves against oxidative stress, using enzymes, such as catalase and superoxide dismutase, small proteins, such as thioredoxin and glutaredoxin, and molecules, such as glutathione. The genetic response of bacteria to oxidative stress is controlled by two major transcriptional regulators (OxyR and SoxRS). ROS destroys various cellular macromolecules resulting in an adaptive oxidative stress response in bacteria to maintain survival against stressors (Kashmiri and Mankar, 2014).

Caffeine has bioactive components that function to reduce fatigue and increase stamina, and is an antioxidant to capture hydroxyl radicals (Liang and David, 2014). Tanin acts to bind metal ions such as Fe (II) and interfere with the Fenton reaction's stages, thus slowing oxidation (Wafa et al., 2016). Tanin cannot increase the chicken's growth. However, it improves the productive aspects of chicken physiology, including inhibiting meat fat oxidation, increasing protein digestibility and feed conversion, improving intestinal health and microbiota biodiversity, and higher capacity to overcome the adverse effects of persistent heat stress (Carrasco et al., 2016). The active ingredient CGA effectively reduces MDA levels through electron transfer, captures free radicals, binds metals, and activates antioxidant enzymes (Hao et al., 2015). Caffeine functions to reduce tissue inflammation through lipid peroxidation markers and pro-inflammatory cytokines in a mouse animal model with alcohol-induced liver damage (Liang and David, 2014).

Phenolic compounds (such as flavonoids, phenylpropanoids, phenolic acids, and tannins) are essential components having biological activities. Polyphenols are generally of chemical patterns; the phenolic group reacts as a hydrogen donor and neutralizes free radicals (Hossain et al., 2014). The CGA consists of an ester family formed between transcinnamic acids, such as caffeic acid and ferulic acid, with quinic acid. Robusta coffee contains between 70 and $300 \mathrm{mg}$ CGA. CGA is a polyphenol with antioxidant activity, and 5-CQA is a strong hydroxyl radical catcher (Liang and David, 2014).

The results of ileum morphometry analysis are presented in Table 2. This research showed that the villus length, villus width, surface area, and depth of the crypts in the negative control are larger than the positive control. Chickens in the treatment group T1 have higher and broader villi than other treatment groups. The length of the ileum villi in the negative control group was higher than the positive control group. There were improvements in the villi length of chickens in the $\mathrm{T} 1$, T2, and T3 treatment groups compared to the positive control. Optimal villi length was found in T1 compared to $\mathrm{T} 2$ and $\mathrm{T} 3$. This finding suggests that the lower the coffee extract dose, the more extended the intestinal villi. Villi help increase food absorption, and the intestinal villi's length is an indicator of intestinal function. The longer the intestinal villi, the better the mucus secretion and absorption of nutrition from the entire small intestine will be (Prakatur et al., 2019). Indeed, in this study, treatment groups T1, T2, and T3 showed weight gain compared to the positive control group. In healthy chickens, the gut bacteria can regulate the host immune defenses, stimulation of mucin production, and proliferation of epithelial cells. In the distal ileum, bacterial density and metabolic activity increase with decreasing digestive rate and weakening digestive fluid's antibacterial potential (Moghaddam and Alizadeh-Ghamsari, 2013).

Table 2. Morphometry of ileum intestine.

\begin{tabular}{cccccc}
\hline Treatments & $\begin{array}{c}\text { Villus length } \\
(\boldsymbol{\mu} \mathbf{m})\end{array}$ & $\begin{array}{c}\text { Villus width } \\
(\boldsymbol{\mu m})\end{array}$ & $\begin{array}{c}\text { Surface area }\left(\times \mathbf{1 0}^{-3}\right. \\
\left.\boldsymbol{\mu m}^{\mathbf{2}}\right)\end{array}$ & $\begin{array}{c}\text { Crypth depth } \\
(\boldsymbol{\mu m})\end{array}$ & $\begin{array}{c}\text { Number of goblet } \\
\mathbf{c e l l}\end{array}$ \\
\hline Negative control & 365.03 & 61.63 & $7,063.99$ & 105.87 & 11 \\
Positive control & 331.028 & 40.51 & $4,210.72$ & 61.34 & 7 \\
T1 & $1,227.35$ & 203.49 & $78,422.58$ & 82.19 & 4 \\
T2 & $1,215.77$ & 114.45 & $43,691.49$ & 63.38 & 7 \\
T3 & $1,215.05$ & 176.93 & $67,503.34$ & 80.28 & 8.6 \\
\hline
\end{tabular}


The histopathology observation showed that the positive control group (chickens infected with $S$. enteritidis bacteria) had erosions in the ileum (Figs. $2 b$ and $5 b$ ) compared to the negative controls and treatment groups T1, T2, and T3, as shown in Figs. 1b, 3b and 4b. The negative control and treatment groups T1 and T2 showed normal epithelial arrangement, elongated villi (Figs. 1a, 3a and 4a), and many goblet cells, as shown in Figs. 1b, $3 \mathrm{~b}$ and $4 \mathrm{~b}$. While in the T3 treatment group, epithelial erosion and short vili resembling that of positive control treatment was observed (Figs 2a and 5a).

Deeper intestinal villi crypts indicate rapid tissue metabolism for repairing and regeneration of intestinal villi so that nutrients can be absorbed quickly. Short intestinal villi are associated with fewer absorption cells and more secretory cells (Prakatur et al., 2019). The number of goblet cells was found higher in negative control compared to other treatments. Secretory cells contribute to the secretion of mucin. Changes in the amount or composition of mucin from the intestinal mucosal surface can reduce the absorption of nutrients and increase the amount of energy needed to maintain intestinal function. The chicken gastrointestinal epithelium is the first line of host intestinal defense and affects nutrient digestion and absorption. The epithelium is coated with a mucous layer that protects the intestine's absorption surface area against harmful microorganisms and acts as a lubricant and transporter between luminal and brush border contents. The mucous layer contains mucin. Mucin is produced and secreted by goblet cells in intestinal epithelial tissue. Under normal physiological conditions, mucin secretion is needed to complete and maintain the intestinal mucous layer's thickness because this layer is often exfoliated by intestinal movements, chemical compounds, and microbes. Various factors such as microbial colonization in the intestine can affect the production, secretion, and mucin composition (Apajalahti and Vienola, 2016).

The intestinal epithelium is continuously exposed to various types of stressor substances, such as feed toxins, that are potentially dangerous and act as a selective barrier between the tissue and the luminal environment of GIT. Furthermore, infectious agents can induce free radicals resulting in imbalance redox reactions. This stress can negatively affect the chicken GIT component's balance, ultimately affecting poultry's health and productivity status (Mishra and Jha, 2019). The relationship between digestible food, digestive enzymes, and bile salts and the contact time between digested particles and absorption surfaces tend to affect the absorption of energy by chickens (Souto et al., 2011). The organic acid content in coffee does not affect weight gain and poultry feed intake, even though it can increase intestinal villi in poultry. Organic acids can also increase the intestine's normal flora and reduce pathogenic bacteria such as Salmonella, thereby reducing inflammatory reactions in the intestinal mucosa (Mohamed et al., 2014).
Alkaloids have various biological effects, such as anti vomit, anti-cholinergic, antitumor, diuretic, sympathomimetic, antiviral, antihypertensive, hypnoanalgesic, antidepressant, mio-relaxant, anti-tussigen, antimicrobial, and anti-inflammatory (Omojate et al., 2014). Tannin is used as an antiseptic because it has a phenolic group. In Ayurveda, herbs with tannin formulations have been used to treat enteric diseases such as diarrhea (Bonelli et al., 2018). In the positive control group, chickens experienced diarrhea, while in the negative control and all treatment groups (T1, T2, and T3), chickens did not experience diarrhea. The antimicrobial mechanisms of tannins are through inhibition of microbial enzyme activity, inhibition of oxidative phosphorylation and ion metabolism, and reducing the availability of essential ions for microorganism's metabolism (Wafa et al., 2016). Other tannin mechanisms as antimicrobials are through inhibition of intestinal bacteria's growth (Videnska et al., 2013), stimulation of the immune system, and modulation of intestinal microbiota (Barboza et al., 2018). Some authors argue that low tannin concentrations can increase feed palatability, thus increasing monogastric animal performance by stimulating feed intake (Carrasco et al., 2016).

Based on histopathological examination, the T3 group showed intestinal epithelial damage characterized by inflammatory cells. In total inflammation, leukocytes in all treatment groups showed no significant difference $(p>0.05)$. However, the number of leukocyte cells of the T3 group was higher than the other treatment groups (unpublished data). Bacterial cell infections cause inflammation in the GIT. Activated phagocytic cell defense will then migrate towards the inflammation area and produces anti-inflammatory metabolism such as proteases, ROS, nitric oxide radicals, and bacterial siderophores. Some antimicrobial products function as pathogenic inactivator. Inflamed cells damage the intestinal epithelium integrity and cause electrolyte release that clinically manifests as diarrhea (Omojate et al., 2014). Inflammation is a protective response of the body against harmful agents such as microorganisms or cell damage (Omojate et al., 2014). CGA functions as an anti-inflammatory agent by decreasing proinflammatory cytokines IL-8 and IL-6 in Caco-2 cells, TNF- $\alpha$, L.P.S., IL-1 $\beta$, and IFN- $Y$ (Liang and Kitts, 2015).

\section{Conclusion}

Green Coffea robusta could potentially increase the antioxidant status and reduce inflammation in the small intestine of layer chickens.

\section{Acknowledgments}

The authors thank the Commodity Research Universities in 2018, the Ministry of Research and Technology, and the University of Brawijaya, Laboratory of Physiology, Faculty of Science and the Laboratory of Pathology and 
Pharmacology, University of Brawijaya, for providing facilities for testing the samples.

\section{Conflict of interest}

The authors declare that there is no conflict of interest.

\section{Authors' contributions}

DR and LER received research funding, research concept, and design. DQS, IAA, and DP: collection and data assembly. FSP, DQS, and AKA: data analysis and interpretation, writing the article, and final approval of the article. IAA and DQS conducted research. All team members wrote and revised this article.

\section{References}

Afshordi, R., Maryam, Z.J., Ali, S., Mohammad, R.P., Saboor-Yaraghi, A.A., Farzaneh, A.H. and Parisa, S. 2016. Oxidative damage caused by common foodborne pathogenic bacteria in egg yolk. Int. J. Enteric Pathog. 4(1), e29123; doi:10.17795/ ijep29123.

Apajalahti, J. and Vienola, K. 2016. Interaction between chicken intestinal microbiota and protein digestion. Anim. Feed Sci. Technol. 221, 323-330. https://doi. org/10.1016/j.anifeedsci.2016.05.004

Aprioku, J.S. 2013. Pharmacology of free radicals and the impact of reactive oxygen species on the testis. J. Reprod. Infertil. 14(4), 158-172; doi:10.1186/ s12917-018-1549-2.

Barboza, J.N., Filho, C.S.M.B., Silva, R.O., Medeiros, J.V.R. and De Sousa, D.P. 2018. An overview on the anti-inflammatory potential and antioxidant profile of eugenol. Oxid Med Cell Longev. 2018, 3957262; doi:10.1155/2018/3957262.

Bonelli, F., Turini, L., Sarri, G., Serra, A., Buccioni, A. and Mele, M. 2018. Oral administration of chestnut tannins to reduce the duration of neonatal calf diarrhea. BMC Vet. Res. 14(1), 227; doi:10.1186/ s12917-018-1549-2.

Carrasco, J.M.D., Redondo, M., Redondo, E.A., Dominguez, J.E., Chacana, A.P. and Miyakawa, M.E.F. 2016. Use of plant extracts as an effective manner to control clostridium perfringens induced necrotic enteritis in poultry. Biomed. Res. Int. 2016, 3278359; doi:10.1155/2016/3278359.

Dias, R.C.E. and De, T.B.M. 2015. Discrimination between Arabica and Robusta Coffees using hydrosoluble compounds: is the efficiency of the parameters dependent on the roast degree. Beverages. 1, 127-139; doi:10.3390/ beverages1030127.

Gayathiri, E., Bharathi, B. and Priya, K. 2018. Study of the enumeration of twelve clinical important bacterial populations at 0.5 mcfarland standard. Int. J. Creat. Res. Thoughts (IJCRT). 6(2), 880-893.

Hao, M.L., Pan, N., Zhang, Q.H. and Wang, X.H. 2015. Therapeutic efficacy of chlorogenic acid on cadmium-induced oxidative neuropathy in a murine model. Exp. Ther. Med. 9(5), 1887-1894; doi:10.3892/etm.2015.2367.
Hossain, H., Al-Mansur, A., Akter, S., Sara, U., Ahmed, R. and Jahangir, A.A. 2014. Evaluation of antiinflammatory activity and total tannin content from the leaves of bacopa monnieri (Linn.). Int. J. Pharm. Sci. Res. 5, 1246-1252.

Joseph, N., Assonfack, F.R.M., Chelea, M., Djiele, N.P. and Ndelo, J. 2016. Evaluation of the antimicrobial activity of tannin extracted from the barks of Erythrophleum guineensis (Caesalpiniaceae). J. Pharm. Phytochem. 5(4), 287-291.

Kashmiri, Z.N. and Mankar, S.A. 2014. Free radicals and oxidative stress in bacteria. Int. J. Curr. Microbiol. App. Sci. 3(9), 34-40.

Khan, C.M.A. 2014. The dynamic interactions between salmonella and themicrobiota, within the challenging Niche of the gastrointestinal tract. Int. Sch. Res. Not. 2014, 846049; doi:10.1155/2014/846049.

Kinuthia, C., Marcus, F. and Oliver, B.B.M. 2017. LCMS analysis of biochemical composition in different Kenyan coffee classifications. Food Sci. Qual. Manag. 69, 44-52.

Liang, N. and David, D.K. 2014. Antioxidant property of coffee components: assessment of methods that define mechanisms of action. Molecules. 19, 19180-19208; doi:10.3390/molecules191119180

Liang, N. and Kitts, D.D. 2015. Role of chlorogenic acids in controlling oxidative and inflammatory stress conditions. Nutrients. 8(1), 16; doi:10.3390/ molecules191119180.

Lobo, V., Patil, A., Phatak, A., Chandra, N., Mishra, B. and Jha, R. 2010. Free radicals, antioxidants and functional foods: Impact on human health. Pharmacogn. Rev. 4(8), 118-126. https://doi. org/10.4103/0973-7847.70902

Martinez-Tome, M., Jime' nez-Monreal, A.M., Garcí aJime' nez, L., Almela, L., Garcı' a-Diz, L., MariscalArcas, M. and Murcia, M.A. 2011. Assessment of antimicrobial activity of coffee brewed in threedifferent ways from different origins. Eur. Food Res. Technol. 233, 497; doi:10.1007/s00217011-1539-0.

Mir, M.A., Kajal, P., Uzma, T. and Ekata, K. 2016. Estimation of alkaloid, saponin and flavonoid, content in various extracts of Crocus sativa. J. Med. Plants Stud. 4(5), 171-174.

Mishra, B. and Jha, R. 2019. Oxidative stress in poultry gut: potential challenges and interventions. Front. Vet. Sci. 6, 60; doi:10.3389/fvets.2019.00060.

Moghaddam, H.N. and Alizadeh-Ghamsari, A.H. 2013. Improved performance and small intestinal development of broiler chickens by dietary L-glutamine supplementation. J. Appl. Anim. Res. 41(1), 1-7. https://doi.org/10.1080/09712119.2012 .738214

Mohamed, M.A., El-Daly, E.F., El-Azeem, N.A.B., Amani, W.S. and Hassan, H.M.A. 2014. Growth performance and histological canges in ileum and immune related organs of broiler fed organic acids 
or antibiotic growth promoter. Int. J. Poult. Sci. 13(10), 602-610; doi:10.3923/ijps.2014.602.610

Omojate, G.C., Enwa, F.O., Jewo, A. and Eze, C. 2014. Mechanisms of antimicrobial actions of phytochemicals against enteric pathogens. J. Pharm. Chem. Biol. Sci. 2(2), 77-85.

Pham-Huy, L.A., He, H. and Pham-Huy, C. 2008. Free radicals, antioxidants in disease and health. Int. J. Biomed. Sci. 4(2), 89-96.

Prakatur, I., Maja, M., Mirela, P., Ksenija, M., Valerija, B., Ivan, M. and Matija, D. 2019. Intestinal morphology in broiler chickens supplemented with propolis and bee pollen. Animals (Basel). 9(6), 301; doi:10.3390/ani9060301.

Qosimah, D., Rosyidi, D., Radiati, L.E., Amri, I.A., Prasetyo, D., Permata, F.S., Beltran, M.A.G., Aulanni'am, A dan Anisa, A.K. 2020. Immunomodulator effect of robusta lampung coffee extract (Coffee Canephora Var Robusta) in layer chicken infected with Salmonella enteritidis bacteria. Indones J Anim Sci. 30(1), 69-79.

Souto, A.L., Tavares, J.F, Sobral da Silva, M., De Fátima Formiga Melo Diniz, M., De Athayde-Filho, F.S. and Filho, J.M.B. 2011. Anti-inflammatory activity of alkaloids: an update from 2000 to 2010. Molecules. 16(10), 8515-8534; doi:10.3390/ molecules16108515.

Videnska, P., Frantisek, S., Hana, H., Marcela, F. and Ivan, R. 2013. Influence of Salmonella enterica serovar Enteritidis infection on the composition of chicken cecal microbiota. BMC Vet. Res. 9, 140; doi:10.1186/1746-6148-9-140.

Wafa, N., Sofianeb, G. and Mouhamed, K. 2016. The antioxidant and antimicrobial activities of flavonoids and tannins extracted from Phlomis bovei De Noé. Euro. J. Exp. Biol. 6(3), 55-61. 